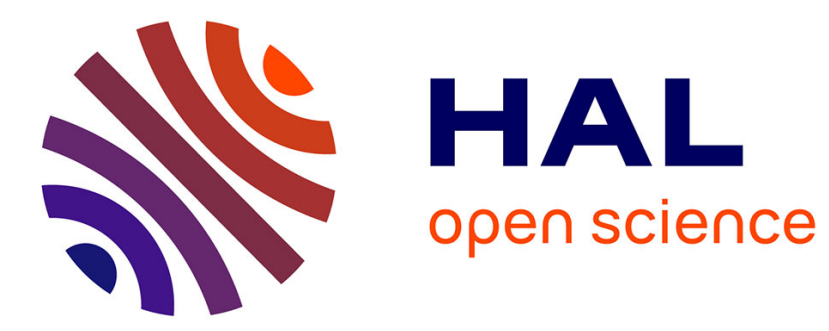

\title{
Qualité de la justice administrative et contentieux des étrangers
}

Danièle Lochak

\section{To cite this version:}

Danièle Lochak. Qualité de la justice administrative et contentieux des étrangers. Revue française d'administration publique, 2016, 159, pp.701-713. hal-01671443

\section{HAL Id: hal-01671443 \\ https://hal.parisnanterre.fr/hal-01671443}

Submitted on 22 Dec 2017

HAL is a multi-disciplinary open access archive for the deposit and dissemination of scientific research documents, whether they are published or not. The documents may come from teaching and research institutions in France or abroad, or from public or private research centers.
L'archive ouverte pluridisciplinaire HAL, est destinée au dépôt et à la diffusion de documents scientifiques de niveau recherche, publiés ou non, émanant des établissements d'enseignement et de recherche français ou étrangers, des laboratoires publics ou privés. 


\title{
Qualité de la justice administrative et contentieux des étrangers*
}

\author{
par Danièle Lochak \\ Professeur émérite de l'Université Paris Ouest - Nanterre La Défense
}

Article paru dans la Revue française d'administration publique, $\mathrm{n}^{\circ}$ 159/2016, pp. 701-713

Résumé : Le contentieux des étrangers est devenu un contentieux de masse. La capacité de la justice administrative à "maîtriser les flux " sans augmentation des "stocks ", devient ici le critère essentiel d'évaluation de la "qualité » de la justice administrative. La régulation des flux contentieux fait ainsi symboliquement écho à l'objectif de régulation des flux migratoires : dans les deux cas on constate la même obsession des chiffres, à l'aune desquels est mesurée l'efficacité d'une politique ou d'une réforme. Mais cette approche purement quantitative est antinomique avec l'exigence de justice et néglige la fonction protectrice dévolue au juge dans un État de droit.

Mots clés : Contentieux administratif - étrangers - éloignement - séjour

Si l'on évalue la «qualité » d'un service à l'aune de sa capacité à satisfaire les besoins de ses bénéficiaires, comme le suggèrent les initiateurs du projet «Qualijus, la question est alors de savoir si les conditions dans lesquelles est rendue la justice dans le domaine du contentieux des étrangers sont de nature à satisfaire les besoins de cette clientèle spécifique. Nous entendons montrer ici que, même en faisant abstraction des motifs d'insatisfaction subjectifs, résultant de ce que les décisions rendues sont bien plus souvent favorables à l'administration qu'aux requérants, il existe de nombreuses raisons objectives de considérer que la qualité n'est pas au rendez-vous. La situation va même en se dégradant au fur à mesure que se succèdent les réformes qui visent théoriquement à l'améliorer.

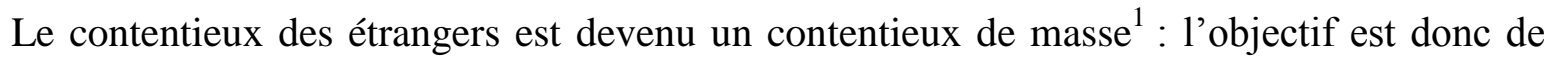
trouver des recettes pour éviter au juge administratif de succomber sous le flux des requêtes. Dans ce contexte, la capacité de la justice administrative à «maîtriser les flux », à traiter les affaires dans les délais impartis, sans augmentation des «stocks », devient le critère essentiel, sinon exclusif, d'évaluation de la «performance » et de la «qualité », au risque d'oublier la fonction protectrice dévolue à la justice dans un État de droit. On assiste en effet à la multiplication de règles dérogatoires, moins protectrices que celles du «droit commun»: délais de saisine raccourcis, procédures accélérées, abandon de la collégialité, appel non suspensif... La régulation des flux contentieux fait ainsi symboliquement écho à l'objectif de régulation des flux migratoires : dans les deux cas on constate la même obsession des chiffres à l'aune desquels sera mesurée l'efficacité d'une politique ou d'une réforme.

* Cet article est une version remaniée et raccourci d'une intervention faite à l'occasion du colloque organisé par l'université de Bretagne occidentale sur «L'efficacité de la justice administrative », le 21 novembre 2014

${ }^{1}$ L'examen des chiffres atteste la place croissante - pour ne pas dire envahissante - qu'occupe le contentieux des étrangers dans l'ensemble du contentieux administratif: en moyenne, toutes juridictions confondues, une affaire sur trois. Mais ce chiffre global n'est pas représentatif. Si on descend plus dans le détail on constate que certains tribunaux traitent plus $30 \%$ d'affaires relevant du contentieux des étrangers - et la proportion atteint $50 \%$ pour le tribunal administratif de Montreuil. Au niveau des cours administratives d'appel la proportion dépasse presque toujours 30\% (et $58 \%$ à Versailles). Pour le Conseil d'État la proportion oscille entre 15 et $20 \%$ mais les affaires jugées incluent le contentieux des mesures réglementaires. 
Cette dérive de la justice des étrangers résulte de la conjonction de deux facteurs : il y a d'abord, évidemment, la volonté du législateur qui, depuis plusieurs décennies désormais, s'évertue à restreindre les garanties accordées aux étrangers qui font l'objet de mesures de refus de séjour ou de reconduite à la frontière pour pouvoir éloigner plus rapidement et plus facilement ceux qui sont en situation irrégulière. Certes, chacune des décisions prises peut faire l'objet d'un recours, parfois même suspensif, et à cet égard les apparences de l'État de droit sont respectées ; mais on met en place des procédures accélérées de façon à ce que la saisine du juge n'entrave pas la procédure d'éloignement - ce qui implique, concrètement, que sa décision intervienne alors que l'étranger est encore en rétention.

Parallèlement, l'orientation répressive de la politique d'immigration accroît inexorablement le nombre de mesures de refus de séjour et d'éloignement prononcées par l'administration et donc le nombre de mesures susceptibles d'être déférées au juge. Et les réformes intervenues au cours des dix dernières années ont amplifié le phénomène alors même qu'elles avaient pour objectif affiché de réduire le nombre des recours.

C'est le cas de la réforme de $2006^{2}$ qui a imaginé de regrouper en un seul et même acte les trois décisions prises jusque-là séparément par le préfet et qui pouvaient donner lieu au minimum à deux recours distincts : contre le refus de séjour, d'une part, contre l'arrêté de reconduite à la frontière et la décision fixant le pays de destination, d'autre part. Désormais le préfet peut accompagner le refus de délivrance ou de renouvellement d'un titre de séjour d'une obligation de quitter le territoire français (OQTF) assortie d'un délai de départ volontaire et fixer dans le même arrêté le pays de destination. Le recours, qui suspend l'éloignement, doit être formé dans un délai de trente jours et le tribunal administratif a trois mois pour statuer. Or non seulement le nouveau dispositif n'a pas enrayé l'inflation contentieuse mais il a suscité un accroissement du taux de recours devant les juridictions du premier degré et du taux d'appel devant les cours administratives d'appel ${ }^{3}$.

Le même phénomène s'est produit avec la réforme suivante, qui visait elle aussi à diminuer le nombre de recours en fusionnant encore plus étroitement le contentieux des refus de séjour et celui de l'éloignement. L'entrée en vigueur de la loi de $2011^{4}$ qui a, d'une part, généralisé la procédure d'urgence en étendant les hypothèses où le juge doit être saisi dans les $48 \mathrm{~h}$, d'autre part reculé dans le temps le moment om l'étranger placé en rétention est présenté au juge des libertés et de la détention (JLD), l'incitant par conséquent à saisir le juge administratif de la décision de placement, a entraîné une explosion du contentieux de l'éloignement des étrangers 5 .

Faute d'avoir la maîtrise de l'amont - le nombre de décisions à contrôler, et à contrôler dans l'urgence -, qui dépend du législateur et de l'administration, les réformateurs de la justice administrative s'efforcent, eux, de réguler les «flux » en aval et imaginent des procédures permettant un traitement plus rapide et allégé de ce contentieux de masse. C'est le second facteur qui contribue à l'instauration de procédures dérogatoires.

Toutes ces réformes, sont présentées, bien entendu, comme préservant les garanties accordées à ces justiciables d'un genre particulier que sont les étrangers. Mais ces garanties ne sont pas réellement protectrices, même si elles permettent, ponctuellement, d'obtenir l'annulation d'une mesure illégale. Car on ne peut rendre une justice de qualité lorsque le rendement est la considération primordiale. L'efficacité appréciée de façon purement quantitative est antinomique avec l'exigence de justice.

\footnotetext{
${ }^{2}$ Loi « Sarkozy » du 24 juillet 2006 relative à l'immigration et à l'intégration.

${ }^{3}$ Rapport de la Commission Mazeaud : «Pour une politique des migrations transparente, simple et solidaire », La Documentation française, 2008, p. 61.

${ }^{4}$ Loi "Besson" du 16 juin 2011 relative à l'immigration, à l'intégration et à la nationalité.

${ }^{5}$ Le dispositif de contrôle sur la rétention a été modifié par la loi du 7 mars 2016, comme on le rappelle plus loin.
} 


\section{L'allègement des obstacles juridictionnels à l'éloignement pour réguler les flux migratoires.}

Ce qui frappe, c'est le contraste entre l'existence de voies de recours, souvent même dotées d'un effet suspensif, contre chacune des décisions prises par l'administration, et la faiblesse des garanties concrètes qu'elles offrent aux requérants. L'accès au juge est entravé par la brièveté des délais de recours, les conditions d'un jugement équitable sont hypothéquées par les difficultés rencontrées pour obtenir une assistance juridique et par les délais très brefs imposés au juge pour statuer. Car le principal souci du législateur est que le passage par la case juridictionnelle ne constitue pas un obstacle à l'éloignement.

\section{Les entraves à l'accès au juge : le spectre du déni de justice}

Non seulement les entraves à l'accès au juge se sont accentuées au fil des réformes mais les conséquences en sont plus graves puisque, aujourd'hui, les procédures d'urgence, et donc l'obligation de déposer un recours dans des délais très brefs, ne s'appliquent pas uniquement aux mesures d'éloignement mais aussi à une grande partie des décisions relatives au séjour celles qui sont accompagnées d'une OQTF sans délai de départ volontaire.

C'est la loi du 10 janvier 1990 (art. 22 bis de l'ordonnance de 1945), qui a pour la première fois instauré un recours suspensif contre les arrêtés de reconduite à la frontière : il devait être introduit dans les 24 heures pour être jugé dans les 48 heures (sachant que la rétention, à l'époque, ne pouvait excéder sept jours). Le nombre de requêtes rejetées comme tardives a prouvé que la protection découlant du caractère suspensif du recours était trop souvent privée d'effet par l'impossibilité de le former en temps utile ${ }^{6}$.

La loi de 1998 a porté le délai de recours contre la mesure d'éloignement à 48 heures lorsqu'elle était remise en mains propres et à sept jours lorsqu'elle était notifiée par voie postale. Mais, compte tenu des obstacles que rencontre un étranger sans papiers pour se faire remettre un pli recommandé à la poste, il arrivait fréquemment que le délai expire sans que le recours puisse être intenté : la mesure, légale ou non, était donc exécutoire et aucun recours ne pouvait plus être formé à son encontre. Mais, à l'époque, cette procédure d'urgence ne concernait que les mesures d'éloignement. Pour contester le refus de délivrance ou de renouvellement d'un titre de séjour, l'étranger disposait du délai de droit commun de deux mois et le recours administratif suspendait ce délai ; le recours pouvait être accompagné d'une demande de sursis à exécution et, après la réforme de 2000, d'un référé-suspension.

La loi Sarkozy du 24 juillet 2006 a modifié ce dispositif. Jusqu'alors, en cas de refus de délivrance ou de renouvellement d'un titre de séjour, l'administration assortissait sa décision d'une invitation à quitter le territoire dans un délai d'un mois ; passé ce délai, elle avait la possibilité de prendre un arrêté de reconduite à la frontière qui, notifié généralement par voie postale, n'était en pratique jamais exécuté. La loi a donc prévu que le refus de séjour pourrait être assorti d'une OQTF, et que cette OQTF deviendrait exécutoire à l'expiration du délai d'un mois, l'étranger pouvant donc être éloigné sans qu'il y ait besoin de prendre une seconde décision. Procédure plus simple, et surtout plus expéditive vue du côté de l'administration ; moins protectrice vue du côté de l'étranger qui n'a plus qu'un mois au lieu de deux pour attaquer, par un même recours, la décision de refus de séjour et l'obligation de quitter le territoire français, sachant que ce délai, de surcroît, n'est plus prorogé par un recours administratif préalable. Et même si ce recours est suspensif de l'exécution de l'OQTF, l'administration peut néanmoins, à l'expiration du délai d'un mois, placer l'étranger en rétention, le tribunal devant alors statuer dans les 72 heures sur l'OQTF.

\footnotetext{
${ }^{6}$ Par exemple : parce que le délai était écoulé au moment où le pli recommandé était retiré à la poste, parce que le cachet de la poste, contrairement à la formule consacrée, ne faisait pas foi, ou encore que les samedis et dimanches n'interrompaient pas le délai...
} 
L'entrée en vigueur de la loi Besson du 16 juin 2011 a réduit encore la portée du délai « de droit commun » : en théorie l'étranger a deux mois pour attaquer un refus de séjour « sec », mais en pratique les refus de séjour sont systématiquement accompagnés d'une OQTF. Le délai de recours n'est plus alors que de trente jours (art. L. 512-1-I du Ceseda) pour demander, par une même requête, l'annulation de la décision relative au séjour, de l'OQTF, le cas échéant de la décision fixant le pays de destination et de l'interdiction de retour sur le territoire français si elle a été prononcée. Et ce délai n'est pas prorogé par un recours administratif (art. R. 776-5I du CJA). La loi du 7 mars 2016 relative au droit des étrangers en France a resserré encore les délais de recours qui passent de trente à quinze jours dans les cas où l'OQTF n'accompagne pas un refus de séjour, notamment si l'étranger est entré irrégulièrement sur le territoire, s'il s'est maintenu en France au-delà de la durée de validité de son visa ou de son titre de séjour dont il n'a pas demandé le renouvellement, enfin et surtout s'il a été « débouté » de sa demande d'asile.

Mais si le préfet n'a pas accordé de délai de départ volontaire ${ }^{7}$ et si l'OQTF est donc immédiatement exécutoire, l'intéressé n'a plus que 48 heures pour déposer son recours et demander l'annulation de toutes les décisions précitées plus le refus d'accorder un délai de départ volontaire, soit cinq au total (art. L. 512-1-II).

Enfin, si l'intéressé a été placé en rétention ou assigné à résidence, il dispose là encore de 48 heures pour demander au juge administratif l'annulation de l'OQTF, éventuellement de l'interdiction de retour, de la décision fixant le pays de destination et de l'assignation à résidence (art. L. 512-1-III). Il peut éventuellement compter sur l'association présente dans le centre de rétention pour l'aider à rédiger son recours, à condition que ce soit pendant les jours et les heures ouvrables.

Comment former ces recours dans le délai imparti sans une assistance juridique solide, même si des assouplissements sont prévus qui permettent de ne déposer qu'une requête sommaire et de la compléter en soulevant des moyens nouveaux jusqu'à l'audience (art. R. 776-5-II du Code de justice administrative) ? L'étranger placé en rétention n'a le plus souvent de contact avec un avocat qu'au moment où il est présenté au juge des libertés et de la détention : désormais à nouveau au bout de 48 heures $^{8}$, donc très tardivement - et à condition qu'il n'ait pas été éloigné dans l'intervalle. Il peut éventuellement compter, pour rédiger et transmettre son recours, sur l'association présente dans le centre de rétention. Mais cette présence n'est assurée que pendant les jours et les heures ouvrables. Et comme le délai de 48 heures est un délai dit «d'heure à heure », qui n'est pas prorogeable en raison des week-ends et des jours fériés, cela veut dire qu'un étranger placé en rétention un vendredi soir ou un samedi matin ne pourra compter sur aucune aide pour rédiger et transmettre son recours.

Lorsque la personne est laissée libre ou assignée à résidence, la situation peut s'avérer paradoxalement plus difficile encore puisque les associations et cabinets d'avocats ne reçoivent pas pendant les week-end et que les permanences d'avocats d'office ne sont ouvertes qu'aux personnes privées de liberté présentées devant un magistrat au cours du week-end.

\section{Une justice équitable?}

Si la brièveté des délais risque de faire obstacle à la saisine du juge, les procédures d'urgence peuvent aussi empêcher, lorsque le recours a été intenté, que la cause soit entendue « équitablement ». L'urgence entrave le travail des avocats et des associations qui assistent

\footnotetext{
${ }^{7}$ Le délai de départ volontaire peut être refusé dans une série d'hypothèses : en cas de menace pour l'ordre public, si la demande de titre de séjour était manifestement infondée ou frauduleuse ou s'il existe un risque que l'étranger se soustraie à l'obligation de quitter le territoire (art. L. 511-1-II al. 2 du Ceseda).

${ }^{8}$ Sous l'empire de la loi Besson, l'étranger n'était plus présenté au juge des libertés et de la détention qu'au terme d'un délai de cinq jours, soit bien après l'expiration du délai de recours contre les mesures prises par l'administration. La loi du 7 mars 2016 a prévu qu'à compter du 1er novembre 2016 le JLD devrait à nouveau être saisi au plus tard au bout de $48 \mathrm{~h}$, l'étranger ayant de son côté la possibilité de contester devant lui la décision de placement en rétention.
} 
l'étranger, mais rend aussi plus délicate la tâche des juges, obligés de statuer rapidement et à la chaîne sur des dossiers qui n'ont pas été préparés comme ils auraient dû l'être.

Certes, les étrangers ont droit à l'aide juridictionnelle dans la plupart des hypothèses où ils font l'objet d'une mesure d'éloignement ou de privation de liberté. Mais l'exercice des droits de la défense subit néanmoins des entraves manifestes.

On le constate, en premier lieu, lorsque l'étranger maintenu en zone d'attente ${ }^{9}$ veut contester le refus d'accès au territoire au titre de l'asile. Depuis $2007^{10}$, il dispose à cette fin d'un recours suspensif qui doit être introduit dans les 48 heures et sera jugé dans les 72 heures (art. L. 213-9 du Ceseda). Mais si les textes prévoient que toute personne maintenue en zone d'attente peut contacter l'avocat de son choix, les étrangers n'ont que rarement les moyens financiers de recourir aux services d'un avocat choisi par eux ; or il n'y a pas de permanence d'avocats en zone d'attente et aucune assistance juridique gratuite et systématique n'est organisée. L'étranger se retrouve donc le plus souvent seul pour préparer dans un délai très bref une requête qui doit être rédigée en français et motivée en droit et en fait. C'est seulement au stade de l'audience qu'il sera représenté par un avocat de permanence, mais celui-ci n'aura pris connaissance du dossier que le jour même et n'aura pu s'entretenir avec l'intéressé que quelques minutes ${ }^{11}$.

Lorsque l'étranger est sous le coup d'une mesure d'éloignement et placé en rétention, il a droit également à l'assistance d'un avocat commis d'office, qui est en général un avocat de permanence ; mais il est là encore difficile à celui-ci d'assurer une défense efficace dans un délai aussi court, alors qu'il n'a eu connaissance du dossier qu'au dernier moment.

La procédure d'urgence, qui suppose la brièveté des délais de recours en amont, implique le raccourcissement des délais de jugement en aval. L'objectif poursuivi est d'accélérer la procédure d'éloignement mais aussi de faire en sorte que le juge rende sa décision pendant que l'étranger est encore en rétention ou en zone d'attente, autrement dit à la disposition de l'administration: la rapidité est ici le corollaire du caractère suspensif du recours puisque l'étranger ne peut être éloigné, si le juge a été saisi, qu'après que celui-ci a statué.

Dans le cas d'une requête en annulation contre une décision de refus de séjour accompagnée d'une OQTF avec délai de départ volontaire, le juge avait, jusqu'à l'entrée en vigueur de la loi du 7 mars 2016, trois mois pour statuer en formation collégiale avec conclusions du rapporteur public, si toutefois celui-ci n'est pas dispensé de les prononcer (art. L. 512-1-I du Ceseda et art. R. 776-13 du CJA). Désormais, dans les cas rappelés plus haut où le requérant n'a lui-même que quinze jours pour intenter un recours ${ }^{12}$, l'audience se déroule devant un juge unique qui n'a plus que six semaines pour statuer. Dans le cas d'une OQTF sans délai de départ volontaire, le tribunal, statuant en formation collégiale, a également trois mois pour statuer (art. L. 512-1-II du Ceseda) - ce qui, soit dit en passant, conduit à s'interroger sur le sens qu'a l'obligation imposée à l'intéressé de déposer son recours dans les 48 heures, sinon d'amoindrir ses chances de pouvoir faire ce recours... Mais il est de toutes façons très rare que l'étranger ne

\footnotetext{
${ }^{9}$ Le placement en zone d'attente concerne les personnes à qui l'accès au territoire est refusé ou qui demandent l'asile à la frontière, alors que le placement en rétention concerne les personnes présentes sur le territoire et sous le coup d'une mesure d'éloignement.

${ }^{10} \mathrm{La}$ loi du 20 novembre 2007 a prévu ce recours à la suite de la condamnation de la France par la Cour européenne des droits de l'homme. La Cour a jugé qu'il y avait eu violation du droit à un recours effectif garanti par l'article 13 combiné avec l'article 3, puisque le requérant, demandeur d'asile, invoquait les risques encourus en cas de refoulement vers l'Erythrée (Cour EDH, G ${ }^{\text {de }}$ Chambre, 26 avril 2007, Gebremedhin c. France).

${ }^{11}$ Les rapports de l'Anafé (Association nationale pour l'assistance aux frontières des étrangers), qu'on peut télécharger sur le site de l'association, fournissent une description précise et documentée de ces difficultés.

${ }^{12} \mathrm{Ce}$ sont en gros les cas où l'OQTF n'accompagne pas un refus de séjour, ce qui correspond par exemple à l'hypothèse où l'étranger est entré irrégulièrement sur le territoire, où il s'est maintenu en France au-delà de la durée de validité de son visa ou de son titre de séjour dont il n'a pas demandé le renouvellement, enfin et surtout où il a été « débouté » de sa demande d'asile.
} 
soit pas, dans ce cas, simultanément placé en rétention, ce qui déclenche la procédure d'urgence proprement dite.

La procédure d'urgence s'applique en effet dès l'instant où l'étranger est placé en rétention ou assigné à résidence, ce qui peut se produire soit immédiatement, dans le cas d'une OQTF sans délai de départ volontaire, soit une fois le délai de départ volontaire expiré. Dans ce cas, le juge doit rendre sa décision dans les trois jours, seul, et sans conclusions d'un rapporteur public (art. L. 512-1-III du Ceseda et art. R. 776-21 du CJA).

C'est ce même délai de 72 heures qui est imparti au juge pour statuer sur les recours formés contre le refus d'entrée sur le territoire au titre de l'asile (art. L. 213-9 du Ceseda).

Ces délais de traitement des affaires ne peuvent pas ne pas avoir d'incidence sur la qualité de la justice rendue et ne pas laisser planer un doute sur l'équité des décisions prononcées dans ces conditions. Certes, le requérant est généralement présent à l'audience, il peut être assisté d'un avocat, et l'un comme l'autre peut produire des documents et présenter des observations orales. Mais l'avocat, lorsqu'il s'agit de l'avocat de permanence, connait mal ou pas du tout le dossier qu'il ne découvre que quelques instants avant l'audience, en même temps qu'il rencontre son client pour la première fois ; le délai de 48 heures, surtout pour une personne placée en rétention, est à l'évidence insuffisant pour réunir les preuves d'une présence durable en France, de l'existence de liens personnels ou familiaux ou encore des risques encourus en cas de retour dans le pays d'origine, autant d'éléments qui pourraient conduire, au regard des droits fondamentaux reconnus par la Convention européenne des droits de l'homme, à remettre en cause le bien-fondé des mesures contestées. Le magistrat statue en général sur le champ, il n'y a donc pas de délai de réflexion supplémentaire. Le sort et la vie des gens peuvent ainsi se jouer sur la base d'une procédure bâclée, qu'il s'agisse des étrangers qui se voient refuser l'accès au territoire au titre de l'asile ou de ceux dont l'OQTF se doublera souvent d'une interdiction de retour sur le territoire français. Or ils n'auront pas de seconde chance pour faire valoir leurs droits puisque l'appel n'est pas suspensif. À supposer même qu'elle ait eu le temps de faire appel et que le juge d'appel prenne finalement une décision favorable à son égard, la personne renvoyée, bien que victime de l'exécution d'une décision illégale, aura en pratique les plus grandes difficultés pour obtenir un visa et revenir en France, sans même parler des obstacles financiers.

*

Le vice-président du Conseil d'État remarquait à juste titre que les contentieux de masse ont souvent trait à l'application des droits fondamentaux ou qu'à à tout le moins, ils touchent à des aspects essentiels de la vie des personnes concernées et impliquent « des enjeux immédiats et importants » pour les requérants (Sauvé, 2011-a). On doute que ces considérations aient été prises en compte dans le contentieux des étrangers, lorsqu'il s'est agi de trouver des parades à l'afflux des recours qui convergent vers la juridiction administrative.

\section{L’allègement des procédures juridictionnelles pour réguler les flux contentieux.}

Pour faire face à l'accroissement du nombre des recours, on a privilégié la voie de l'allégement des procédures - ce qui, comme le relève Olivier Lecucq, " comporte des risques non seulement pour les étrangers, mais aussi pour la justice administrative elle- même » (Lecucq, 2012). Pour justifier ce choix, on a fait valoir que les affaires «présentent à juger, dans la grande majorité des espèces, des questions de droit déjà tranchées et des questions de fait assez simples » (Sauvé, 2012). S'il est vrai que les questions de droit soulevées sont le plus souvent simples, ce n'est pas systématiquement le cas ; et surtout, s'agissant du contentieux du séjour et de l'éloignement, les questions de fait supposent une évaluation fine des situations qui gagnerait justement à ne pas être confiée à un juge unique, statuant en urgence et sans conclusions d'un rapporteur public. 
Les réformes de procédure visant à accélérer le traitement du contentieux ont été dénoncées, par des voix issues de la juridiction administrative elle-même, comme le signe d'une " dérive managériale » $(\text { Costa, 2010 })^{13}$ plus préoccupée de chiffres et de rendement que de qualité. De fait, si l'on se réfère à la loi de finances, on constate que, s'agissant des juridictions administratives, les «performances » sont appréciées à l'aune des délais de jugement et que le renforcement de l' «efficience » est mesuré par deux indicateurs strictement quantitatifs : le nombre d'affaires réglées par magistrat et le nombre d'affaires réglées par agent de greffe. La qualité des décisions juridictionnelles, quant à elle, est mesurée... par le taux d'annulation des décisions ${ }^{14}$.

La préoccupation exclusive des délais de jugement et de l'écoulement des stocks ont débouché sur la mise en place de procédures simplifiées - amenuisement de la place de la collégialité, dispense des conclusions - ou carrément expéditives comme le recours aux ordonnances de « tri ».

\section{La disparition programmée de la collégialité}

Ce qui devrait être normalement l'exception est devenu la règle : la proportion de décisions rendues par un juge unique s'élève, grosso modo, à $40 \%$ pour le Conseil d'État, $30 \%$ pour les cours administratives d'appel, $60 \%$ pour les tribunaux administratifs. Cette réalité n'est propre ni à la juridiction administrative ni au contentieux des étrangers. Mais celui-ci, outre qu'il a servi de terrain d'expérimentation en la matière, reste le domaine par excellence du juge unique.

Lorsque le législateur a créé, en 1990, un recours suspensif contre l'arrêté de reconduite à la frontière, il l'a confié à un juge unique. C'est également à un juge unique qu'ont été confiés, en 2006, les recours contre les OQTF lorsqu'elles n'accompagnaient pas un refus de séjour ou lorsque la personne était placée en rétention. Et c'est encore à un juge unique qu'est revenu le soin, sur le fondement de la loi de 2011, dans l'hypothèse fréquente où la personne est placée en rétention, de statuer non seulement sur l'OQTF, sur le pays de retour, sur la décision de placement en rétention ${ }^{15}$, mais aussi, le cas échéant, par voie d'exception, sur le refus de séjour et sur l'interdiction de retour sur le territoire français. Cette extension progressive du domaine de compétence du juge unique est encore confirmée par la loi du 7 mars 2016. Alors que la réforme de 2011 avait laissé subsister la compétence de la formation collégiale pour statuer sur une OQTF lorsque la personne n'est pas placée en rétention, c'est désormais un juge unique qui statuera, dans un délai de six semaines au lieu de trois mois, sur les recours contre une OQTF dans les cas où elle n'accompagne pas un refus de séjour.

L'évolution affecte aussi le contentieux de l'asile. Jusqu'à présent, seul l'étranger qui se voyait refuser l'accès au territoire au titre de l'asile comparaissait devant un juge unique selon le schéma habituel : saisine dans les 48 heures et jugement dans les 72 heures. La loi du 29 juillet 2015 relative à la réforme du droit d'asile a institué un juge unique au sein même de la Cour nationale du droit d'asile pour connaître des recours contre les décisions de l'OFPRA lorsque la personne a été placée en procédure dite « accélérée »- une procédure susceptible de s'appliquer dans un nombre important d'hypothèses ${ }^{16}$. Cette orientation vers un circuit court -

\footnotetext{
${ }^{13}$ L'auteure s'exprime ici en tant que présidente du Syndicat de la juridiction administrative.

${ }^{14}$ Avis présenté au nom de la commission des lois sur le projet de loi de finances pour 2015, Tome V : Juridictions administratives et administrations financières, Doc. Sénat $n^{\circ}$ 114, 20 novembre 2014, p. 13 et s.

${ }^{15}$ La loi du 7 mars 2016 a toutefois ôté au juge administratif la compétence pour statuer sur la décision de placement en rétention : à compter du $1^{\mathrm{er}}$ novembre 2016 cette décision devra être contestée devant le JLD.

${ }^{16}$ L'article L. 723-2 du Ceseda énumère la très longue liste d'hypothèses où l'Office doit ou peut statuer en "procédure accélérée" - nouveau nom de la procédure dite précédemment "prioritaire". Outre les ressortissants originaires de pays considérés comme "sûrs", la procédure peut concerner par exemple le demandeur soupçonné d'avoir menti sur son identité ou sa nationalité, celui dont la demande n'est pas suffisamment pertinente ou dont les déclarations sont manifestement incohérentes et contradictoires ou encore celui qui a refusé de donner ses empreintes digitales, qui a présenté sa demande d'asile tardivement, qui n'a demandé l'asile qu'en vue de faire échec à une mesure d'éloignement, etc.
} 
puisque le juge devra dans ce cas statuer dans un délai de cinq semaines au lieu de cinq mois, désormais, dans la procédure normale - et vers une audience à juge unique a été critiqué par le $\mathrm{HCR}^{17}$ qui a rappelé son attachement à la collégialité : à ses yeux, le jugement par un juge unique ne présente pas les mêmes garanties qu'une formation collégiale - une formation collégiale dans laquelle, rappelons-le, siège un juge assesseur désigné par le HCR, qui n'est donc plus présent.

La généralisation du juge statuant seul permet assurément de désencombrer le prétoire et d'accélérer le traitement des affaires qui ont ainsi une chance d'être jugées dans des délais plus « raisonnables ». Mais à quel prix ? Les garanties auxquelles ont droit les justiciables ne sontelles pas sacrifiées sur l'autel de la productivité et du rendement ? La décision rendue par un juge unique, qui ne bénéficie donc pas de la «confrontation des idées et des perceptions », n'est-elle pas plus fragile ? (Seiller, 2012). Même si l'indépendance et l'impartialité n'ont pas de raison d'être plus mises en doute lorsque le juge statue seul que lorsqu'il statue collégialement, il n'en demeure pas moins que le jugement rendu par un juge seul accroît les risques de disparité de traitement entre les justiciables : tous les avocats peuvent témoigner de l'influence qu'a sur le sort de leur client le juge qui va connaître de son affaire. Ces disparités sont d'autant plus graves que très souvent il n'existe pas de voies de recours efficaces contre les décisions rendues par un juge unique : soit elles ne sont pas susceptibles de recours, soit les recours ne sont pas suspensifs.

\section{La raréfaction des conclusions}

Le juge unique, de surcroît, est bien seul. Initialement, toutes les affaires jugées par un magistrat statuant seul, sauf celles réglées par voie d'ordonnance, bénéficiaient au moins du deuxième regard que constitue l'intervention du rapporteur public. Aujourd'hui, dans les hypothèses - nombreuses, on l'a vu - où l'affaire est jugée selon la procédure d'urgence, il n'y a pas de conclusions.

Mais l'évolution affecte aussi la procédure collégiale, de sorte que l'absence de conclusions est en voie de devenir la norme pour l'ensemble du contentieux des étrangers. On sait que la loi du 17 mai 2011 et le décret d'application du 23 décembre 2011 ont prévu la possibilité pour la formation collégiale ou le magistrat statuant seul de dispenser le rapporteur public de prononcer ses conclusions dans une série de matières, tant en première instance qu'en appel. Parmi celles-ci, énumérées à l'article L. 732-1-1 du code de justice administrative, figurent le contentieux des naturalisations et le contentieux de l'entrée, du séjour et de l'éloignement des étrangers, expulsion exclue.

Cette réforme, selon le vice-président du Conseil d'État, vise à permettre au rapporteur public de se recentrer sur son «cœur de métier» et d'éviter «de se disperser, voire de s'épuiser, sur des dossiers qui posent des questions récurrentes dans un cadre juridique parfaitement déterminé » et ne présentent aucune difficulté sur le plan du raisonnement juridique ou de l'appréciation des faits (Sauvé, 2011-b). Mais comment concilier cette idée du caractère parfois superflu des conclusions du rapporteur public avec la défense et illustration de l'institution à laquelle s'est livré le Conseil d'État devant la Cour européenne des droits de l'homme dans l'affaire Marc-Antoine c. France ${ }^{18}$ ? Le gouvernement français - mais c'est évidemment le Conseil d'État qui tenait la plume - rappelait dans ses observations ${ }^{19}$ «la contribution essentielle du rapporteur public à la qualité de la décision de justice »; il le présentait comme «un rouage essentiel de la collégialité, conçue comme un gage d'exhaustivité dans le traitement des dossiers », qui concourt à éviter les erreurs et les omissions ; il insistait sur «sa contribution à l'équité de la procédure » et sur le fait que son intervention apportait « un gain considérable en termes de contradiction et de transparence de

\footnotetext{
${ }^{17}$ Note du HCR sur le projet de loi relatif à la réforme de l'asile, octobre 2014.

${ }^{18}$ CEDH, 4 juin 2013, Marc-Antoine c. France, Req. ${ }^{\circ}$ 54984/09

${ }^{19}$ Observations initiales du 16 mars 2012.
} 
la procédure »; au point que sa fragilisation constituerait un recul pour les droits des justiciables.

Si vraiment le rapporteur public joue un rôle aussi essentiel au service des justiciables, comment peut-on justifier qu'on se dispense de ses conclusions dans un nombre croissant de contentieux qui concernent en priorité les catégories de justiciables les plus vulnérables? L'argument selon lequel l'absence de conclusions peut être compensée ou même avantageusement suppléée par «une plus grande place laissée au cours de l'audience à l'expression orale des parties » Sauvé, 2011-b) ne convainc guère dans le cas du contentieux des étrangers: l'urgence ne favorise guère l'échange approfondi d'arguments, a fortiori lorsque les requérants doivent s'exprimer dans une langue qu'ils maîtrisent mal ou doivent passer par l'intermédiaire d'un interprète. Plutôt que d'une «revalorisation » du rapporteur public, la réforme conforte l'évolution vers une « justice à deux vitesses » où les contentieux dits « de masse » seront traités avec des garanties juridictionnelles toujours plus réduites, au détriment de l'égalité devant la justice (Costa, 2010).

\section{Le couperet des ordonnances de tri}

Le code de justice administrative prévoit la possibilité pour le juge de statuer seul par voie d'ordonnance, sans conclusions du rapporteur public et sans audience, dans une série d'affaires ne présentant pas de réelles difficultés juridiques telles que les désistements, les requêtes ne relevant manifestement pas de la compétence du juge administratif ou manifestement irrecevables ${ }^{20}$. Mais le décret du 23 décembre 2006 a étendu cette possibilité au rejet des requêtes «ne comportant que des moyens de légalité externe manifestement infondés, des moyens irrecevables, des moyens inopérants ou des moyens qui ne sont assortis que de faits manifestement insusceptibles de venir à leur soutien ou qui ne sont manifestement pas assortis de précisions permettant d'en apprécier le bien-fondé » (art. R. 222-1, $7^{\circ}$ ). Cet ajout non seulement élargit considérablement le champ d'application de la procédure mais en modifie sensiblement la philosophie : il ne s'agit plus de se livrer à un constat objectif mais de «trier » les requêtes sur la base d'une appréciation beaucoup plus subjective.

Devant la CNDA, également, le président ou un président de section peut rejeter par ordonnance les demandes qui ne présentent « aucun élément sérieux susceptible de remettre en cause les motifs de la décision de l'OFPRA » (art. R. 733-4, $5^{\circ}$ du Ceseda). Une possibilité que déplore le HCR pour qui l'appréciation des demandes « implique une appréciation du fond de l'affaire, que l'oralité des débats et la collégialité permettraient de rendre complète, transparente et pleinement effective $»^{21}$.

Aux yeux du vice-président du Conseil d'État, l'extension de la procédure de l'ordonnance aux requêtes manifestement mal fondées procèderait «d'une vision concrète du service rendu qui permet d'éviter aux demandeurs, dont la requête est vouée au rejet, de devoir attendre plusieurs mois, voire plusieurs années, avant d'en être informé » (Sauvé, 2011-a). Vision concrète ou vision angélique? Vision contestable en tous cas, tant le procédé va à l'encontre de tous les principes fondamentaux qui régissent la procédure contentieuse : la collégialité, le contradictoire, la publicité des audiences.

La méconnaissance de ces principes laisse le champ libre à la subjectivité du juge, d'autant qu'il est difficile, au vu d'une requête souvent sommaire, de préjuger de son devenir, le requérant pouvant la compléter en cours d'instance, produire des pièces nouvelles ou soulever des moyens nouveaux. Au-delà, la tentation est grande, dans les juridictions les plus saturées, d'utiliser l'ordonnance de tri comme un moyen de désencombrer les rôles au prix d'une entrave supplémentaire à l'exercice du droit de recours.

En pratique les justiciables et leurs avocats ont l'impression d'une loterie, tant le résultat du tri s'avère aléatoire et peut varier en fonction de la juridiction saisie ou du magistrat qui jugera.

\footnotetext{
${ }^{20}$ Art. R. 122-12 du CJA, en ce qui concerne le Conseil d'Etat, et R. 222-1 pour les tribunaux et les cours.

${ }^{21}$ Dans sa note précitée sur le projet de loi sur l'asile, le HCR dit regretter le maintien de cette procédure.
} 
Certes, une ordonnance de tri peut être contestée, mais seulement par la voie d'un recours en cassation devant le Conseil d'État: ce qui suppose le ministère d'avocat alors que l'aide juridictionnelle n'a guère de chance d'être accordée sur une ordonnance de tri en raison du caractère principalement factuel du litige.

Impuissants à réguler le flux des entrées, les réformateurs de la justice administrative cherchent à tout prix à accroître le flux des sorties par l'allègement des procédures, de façon à préserver les tribunaux administratifs qui ploient sous l'afflux de requêtes. Mais cet allègement se fait au prix du sacrifice des garanties procédurales et d'une moindre qualité des décisions rendues qui en découle (Weber, 2008).

Pour expliquer le développement exponentiel du contentieux des étrangers et l'encombrement des juridictions qui en résulte, certains ont cru pouvoir crier haro sur les avocats et sur les associations ${ }^{22}$. Outre qu'on ne saurait faire grief à quiconque d'utiliser pleinement les moyens de droit qui lui sont reconnus par les textes, rejeter la faute sur les requérants, leurs avocats ou les associations est une façon d'escamoter les véritables causes de l'explosion du contentieux.

Ces causes sont à rechercher en premier lieu dans les choix qui guident la politique d'immigration. On ne s'y attardera pas, sinon pour rappeler que la lutte contre l'immigration irrégulière n'en est qu'une des facettes, la plus visible, l'autre étant la précarisation du droit au séjour des étrangers résidant régulièrement en France et le rétrécissement des voies d'accès régulières au territoire pour ceux qui auraient vocation, sur le fondement des principes internationalement reconnus comme le droit d'asile ou le droit au respect de la vie familiale, à s'y établir. Cette remarque n'est pas hors sujet : elle implique en effet que, parmi les personnes à qui le droit au séjour est refusé et à qui l'on impose de quitter le territoire, nombreuses sont celles qui ont des raisons impérieuses d'y demeurer et pour lesquelles le recours contentieux est véritablement $l a$ dernière chance pour faire obstacle à une mesure qui peut bouleverser leur vie, et pas simplement une « opportunité » à saisir, comme le suggère le rapport précité.

Parmi les éléments du système mis en place au fil des réformes et qui concourent à rendre la voie contentieuse inéluctable, il faut mentionner l'absence de toute autre possibilité de défendre sa cause devant l'administration. La consultation de la commission du titre de séjour est devenue résiduelle et ses avis, facultatifs, ne sont pas nécessairement suivis. Rappelons aussi la disparition du recours gracieux et hiérarchique qui permettaient de régler bon nombre de situations en amont de la saisine du juge : le recours administratif ne prorogeant plus les délais et n'ayant aucune chance d'aboutir avant l'expiration du délai de recours contentieux ramené à trente jours lorsque le refus de séjour s'accompagne d'une OQTF, il est devenu inutile; et dans le cadre de la procédure d'urgence il est tout simplement exclu.

Aussi longtemps, par conséquent, que la réglementation restera ce qu'elle est et que les pratiques préfectorales et policières resteront ce qu'elles sont, il est illusoire d'imaginer faire régresser la charge qui pèse sur la juridiction administrative. Mais il est tout aussi illusoire de penser qu'on peut soulager le juge sans sacrifier les garanties de la défense et le droit à un jugement équitable.

Soumis à des normes de rendement, le juge subit de plus la pression implicite d'un gouvernement qui, anxieux de voir les mesures d'éloignement exécutées, est enclin à considérer l'instance juridictionnelle comme une entrave à l'efficacité de sa politique. En

${ }^{22}$ Le rapport Mazeaud, par exemple, dénonce «l'instrumentalisation de la justice par des tactiques contentieuses, souvent inspirées par des professionnels spécialisés et des groupes militants », qui exploitent la complexité de la législation et les failles qu’elle recèle (Rapport précité, p. 64) 
témoigne la lecture du rapport annuel au Parlement où sont mentionnées, parmi les obstacles à la mise en œuvre des mesures d'éloignement, « les annulations de procédure par la justice pénale ou administrative» avec cette phrase récurrente qui énonce que «les annulations juridictionnelles de procédures d'éloignement ont été à l'origine [en telle année] de $\mathrm{x} \%$ des échecs enregistrés ${ }^{23}$. Curieuse conception du rôle du juge dans un État de droit...

\section{Bibliographie}

Costa, Elise (2010), « Des chiffres sans les lettres. La dérive managériale de la juridiction administrative », AJDA, $\mathrm{n}^{\circ}$ 29, 3 septembre, p. 1623.

Lecucq Olivier (2012), «Le contentieux des étrangers : un contentieux de masse auquel il faut faire face », $A J D A, \mathrm{n}^{\circ} 22,25$ juin, p. 1210.

Sauvé, Jean-Marc (2011-a), allocution de clôture, «Dix années de croissance du contentieux : Quelles réalités ? Quelles réponses ? », RFDA, $\mathrm{n}^{\circ} 4, \mathrm{p} .684$.

Sauvé, Jean-Marc (2011-b), interview, Dalloz Actualité, 6 mars.

Sauvé, Jean-Marc (2012), « Le juge administratif face au défi de l'efficacité. Retour sur les pertinents propos d'un Huron au Palais-Royal et sur la "critique managériale" » $R F D A, \mathrm{n}^{\circ} 4$, $\mathrm{p}$. 613.

Seiller, Bertrand (2012), « Le juge unique », AJDA, $\mathrm{n}^{\circ} 22,25$ juin, p. 1205

Weber, Anne (2008), «Le juge administratif unique, nécessaire à l'efficacité de la justice administrative ? », RFAP, $\mathrm{n}^{\circ} 125$, p. 179.

\footnotetext{
${ }^{23}$ Voir par exemple le rapport sur «Les orientations de la politique d'immigration », La Documentation française, décembre 2007 ou le $9^{\text {e }}$ rapport, intitulé «Les chiffres de la politique de l'immigration et de l'intégration », remis en décembre 2012 et qui porte sur l'année 2011. Dans le $10^{\mathrm{e}}$ rapport, intitulé "Les étrangers en France », qui porte sur l'année 2012, on ne parle plus d'obstacles mais de " sécurité juridique et opérationnelle des éloignements », mais le lien entre la censure juridictionnelle et l'échec est conservé.
} 